\title{
Sarcopenia - Endocrinological and Neurological Aspects
}

\author{
Authors \\ Michaela Katja Stangl', Wolfgang Böcker², Vladimir Chubanov³, Uta Ferrari', Michael Fischereder ${ }^{4}$, Thomas Gudermann³, \\ Eric Hesse ${ }^{5}$, Peter Meinke ${ }^{6}$, Martin Reincke ${ }^{1}$, Nicole Reisch ${ }^{1}$, Maximilian M. Saller ${ }^{2}$, Jochen Seissler ${ }^{1}$, Ralf Schmidmaier ${ }^{1}$, \\ Benedikt Schoser ${ }^{6}$, Cornelia Then ${ }^{1}$, Barbara Thorand ${ }^{7}$, Michael Drey ${ }^{1}$
}

\section{Affiliations}

1 Department of Medicine IV, University Hospital, LMU Munich, Germany

2 Experimental Surgery and Regenerative Medicine (ExperiMed), Department of General, Trauma and Reconstructive Surgery, University Hospital, LMU Munich, Germany

3 Walther Straub Institute of Pharmacology and Toxicology, Faculty of Medicine, LMU Munich, Germany

4 Department of General, Visceral and Transplantation Surgery, University Hospital, LMU Munich, Germany

5 Heisenberg-Group for Molecular Skeletal Biology, Department of Trauma, Hand and Reconstructive Surgery, University Medical Centre Hamburg-Eppendorf, Hamburg, Germany

6 Friedrich Baur Institute at the Department of Neurology, University Hospital, LMU Munich, Germany

7 Institute of Epidemiology, Helmholtz Center Munich, German Research Center for Environmental Health, Munich, Germany

\section{Key words}

sarcopenia, pathomechanisms, muscle, hormones, diabetes, neurodegeneration

received 21.06 .2018

revised 06.08 .2018

accepted 08.08.2018

\section{Bibliography}

DOI https://doi.org/10.1055/a-0672-1007

Published online: 10.9.2018

Exp Clin Endocrinol Diabetes 2019; 127: 8-22

(c) J. A. Barth Verlag in Georg Thieme Verlag KG Stuttgart .

New York

ISSN 0947-7349

\author{
Correspondence \\ Michael Drey \\ Department of Medicine IV \\ University Hospital \\ LMU Munich \\ Ziemssenstrasse 1 \\ 80336 Munich \\ Germany \\ Tel.: +49/89/440052 940, Fax: +49/89/440054931 \\ Michael.Drey@med.uni-muenchen.de
}

\section{ABSTRACT}

Sarcopenia in geriatric patients is often associated with or even caused by changes of the endocrine and nervous system. The multifactorial pathogenesis of sarcopenia and additional multimorbidity in geriatric patients makes it difficult to study distinct pathogenic pathways leading to sarcopenia. Patients suffering from diabetes, Cushing's syndrome, chronic kidney disease, Klinefelter's syndrome or motor neuron diseases, such as amyotrophic lateral sclerosis for example are known to have impaired muscle property and reduced physical performance. These patients are typically younger and suffer from conditions caused by a known molecular disease mechanism and a peculiar sarcopenic phenotype. Therefore, these sequelae can serve as prototypic disease models to study isolated endocrinological and neurodegenerative causes for sarcopenia. This review focuses on diseases whose etiopathogenesis of muscle impairment is known. The idea is to use these diseases as proof of principles to develop a classification algorithm of sarcopenia in the elderly to make a more mechanism-oriented therapy be possible.

\section{Introduction}

Demographic changes and increasing life expectancy worldwide pronounce the importance to understand adjustable risk factors for disability and loss of independence with advancing age. For more than two decades there has been an increasing interest in the role of sarcopenia representing today a geriatric syndrome curtailing active and healthy aging [1]. Already in the early twentieth century, physicians were concerned about the loss of muscle mass in the extremities with old age, but did not have a particular name for this phenomenon for a long time. In the 1980ies, Irvin Rosenberg 
first coined the term sarcopenia from the Greek roots of the word sarx for flesh and penia for loss to describe important changes in the body composition and related functions [2].

In 2010, the European Working Group on Sarcopenia in Older People (EWGSOP) defined age-related sarcopenia as a syndrome characterized by progressive and generalized loss of skeletal muscle mass and loss in muscle strength or muscle function. This condition is associated with a risk of adverse outcomes such as physical disability, poor quality of life and even death [1]. Subsequent definitions of sarcopenia are all based on low muscle mass and reduced muscle strength and/or muscle function [3]. Current diagnosis of sarcopenia is based on low muscle mass measured with dual-energy X-ray absorptiometry (DEXA) or bioelectrical impedance analysis [1], and low gait speed and/or low hand grip strength, measured by an handheld dynamometer [4]. If muscle mass, gait speed and/or hand grip strength are below the age- and gender specific cut-off, the diagnosis is made. A questionnaire for sarcopenia screening (SARC-F) is also available, identifying a decreased physical performance and falls [5]. Depending on the available literature, the prevalence for sarcopenia in the age of $60-70$ years is reported as $5-13 \%$, while the prevalence ranges from $11-50 \%$ in people 80 years and older [1].

A number of potential biomarkers for sarcopenia are under investigation which will be described in more detail below and might lead to new diagnostic tools and potential future targets for the treatment of sarcopenia. Today, the primary treatment of sarcopenia is resistance training which is effective, even in advanced age [6]. This is usually combined with a high protein $(1.2 \mathrm{~g} / \mathrm{kg} / \mathrm{d})$ diet and vitamin D supplementation as muscle protein synthesis is enhanced when consuming leucine-enriched amino acids and vita$\min \mathrm{D}$ [7].

An important step for increased recognition as a disease entity and treatment of patients with sarcopenia was achieved in the US by awarding an ICD-10-CM (M62.84) code in September, 2016 [8] and in Germany in January, 2018 (ICD-10-GM M62.50). This was the most important step leading to an accelerated awareness by physicians. As a consequence this was echoed by the interest of pharmaceutical companies and nutritional industry in developing new drugs to treat sarcopenia.

Nevertheless, the current diagnosis and treatment of sarcopenia is quite unspecific and not based on the underlying etiopathogenesis. This is mainly caused by a lack of knowledge due to the multifactorial genesis of sarcopenia and multimorbidity of the geriatric patient. In this review we will spotlight an idea for bypassing particularly the problem of multimorbidity masking pathological processes of sarcopenia. Distinct diseases that occur in younger, non-geriatric patients leading to a decline in muscle performance represent prototypic disease models for investigating etiopathogenesis of alternative muscle degeneration (> Fig. 1). In the following, we are summarizing ideas about multifactorial etiopathogenic mechanisms of sarcopenia followed by the representation of five human prototypic disease models for sarcopenia in younger sarcopenic patients. Those diseases of either neurological or endocrinological origin have all in common to occur already in non-geriatric patients and lead to an associated sarcopenic phenotype that we will term "secondary sarcopenia". Diseases associated with "secondary sarcopenia" are represented by: diabetes with diabetic sarcopenia, Cushing's syndrome (CS) with sarcopenic obesity, chronic kidney diseases (CKD) with uremic sarcopenia, Klinefelter's syndrome (KS) with hypogonadal sarcopenia and motor neuron disease, presented by amyotrophic lateral sclerosis (ALS) with neurogenic sarcopenia ( $>$ Fig. 2 ). They will serve as human prototypic disease models to study solitary endocrinel and neurodegenerative causes for sarcopenia to elucidate and characterize in detail which pathways might be important for muscle regeneration and could be used therpeutically. Such an approach which links the evidence from those human models of nature with sarcopenia in the elderly might help to develop a classification algorithm and a more mechanism-oriented therapy for affected patients.

\section{General Etiopathophysiological Mechanisms of Sarcopenia}

The etiopathophysiology of sarcopenia is highly complex and multifactorial which hampers the diagnostic approach and leads to an unspecific treatment. There are known risk factors such as malnutrition, anorexia, inflammation, lack of activity, hypovitaminosis D, poor blood flow to muscle, and predisposal genetic and epigenetic aspects leading to sarcopenia [6].

In sum, all these factors may cause a decline in muscle mass, strength, function, and capacity. Thus, insulin resistance seems to be one of the major factors in diabetes causing associated diabetic obesity, glucocorticoid excess seems to be involved in sarcopenic obesity (CS), creatinine and chronic inflammation in CKD and the resulting uremic sarcopenia, low testosterone levels are main reasons for hypogonadal sarcopenia resulting from KS, and finally motor neuron loss triggers the development of neurological sarcopenia (ALS).

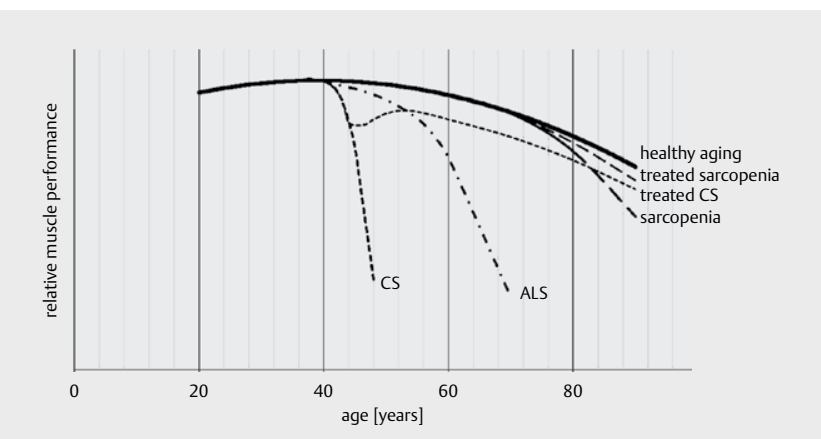

Fig. 1 Age- and disease-related changes in muscle performances. Healthy aging results in a smooth decline of muscle performance starting in the $4^{\text {th }}$ decade of life, whereas sarcopenia is characterized by a stronger decline of muscle performance. Endocrine diseases such as Cushing's syndrome (CS), diabetes, chronic kidney disease and Klinefelter's syndrome, as well as motor neuron diseases, such as amyotrophic lateral sclerosis (ALS) often result in a disease-related "secondary sarcopenia". (Only some of the named diseases are indicated in the figure for a clear arrangement.) These diseases represent prototypic models for sarcopenia and the information about their pathomechanisms, as well as biomarkers and tests for diagnosis and pharmaceutical approaches for their treatment might help to classify sarcopenic patients and help to find a patient-oriented therapy. 
Investigating the specific etiopathogenesis of these pre-ageing diseases will help to decipher what they might contribute to a global pathophysiology of sarcopenia resulting in decline of the total protein biosynthesis, combined with an increase in degradation of specific muscle proteins, mitochondrial dysfunction with reduced energy production, and muscle atrophy and apoptosis via activation of the ubiquitin proteasome system (UPS). Furthermore, an inhibition of satellite cell recruitment and decreased neuromuscular junction formation is most likely [6] (• Fig. 3).

\section{Human Prototypic Disease Models for Geriatric Sarcopenia}

\section{Diabetes and diabetic sarcopenia}

Reduced insulin actions due to insulin resistance and/or depletion seem to be involved in the aggravation of muscle dysfunction. Recent-onset type 2 diabetes (T2D) is associated with sarcopenia in elderly ( $\geq 75$ years), but not in middle-aged or younger subjects
[9]. The older participants in this study possessed a higher insulin resistance, whereas the younger participants predominantly exhibited a reduced insulin secretion capacity, which does not only indicate a different pathological pathway of T2D manifesting earlier or later in life, but also a stronger association of sarcopenia with insulin resistance than with insulin depletion. This assumption is affirmed by further studies showing that insulin resistance is an independent risk factor of decreased skeletal muscle mass [10].

Insulin is an anabolic and metabolic hormone increasing muscle protein synthesis and limiting protein degradation [11]. It induces a pathway involving phosphoinositide3-kinase (PI3K), phosphoinositol triphosphate, protein kinase B/AKT, TBC1 domain family member (TBC1D) 4 and TBC1D1, resulting in the translocation of glucose transporter type 4 (GLUT4) containing vesicles to the plasma membrane [12] and the dis-inhibition of glycogen synthesis by phosphorylation of glycogen synthase kinase 3. AKT also induces the activation of mTOR (mechanistic target of rapamycin), which promotes protein synthesis, and inhibits forkhead box protein O (FOXO) activity, thus reducing the expression of E3 ubiquitin ligases that mediate atrophy [13] ( Fig. 3). AKT overexpression

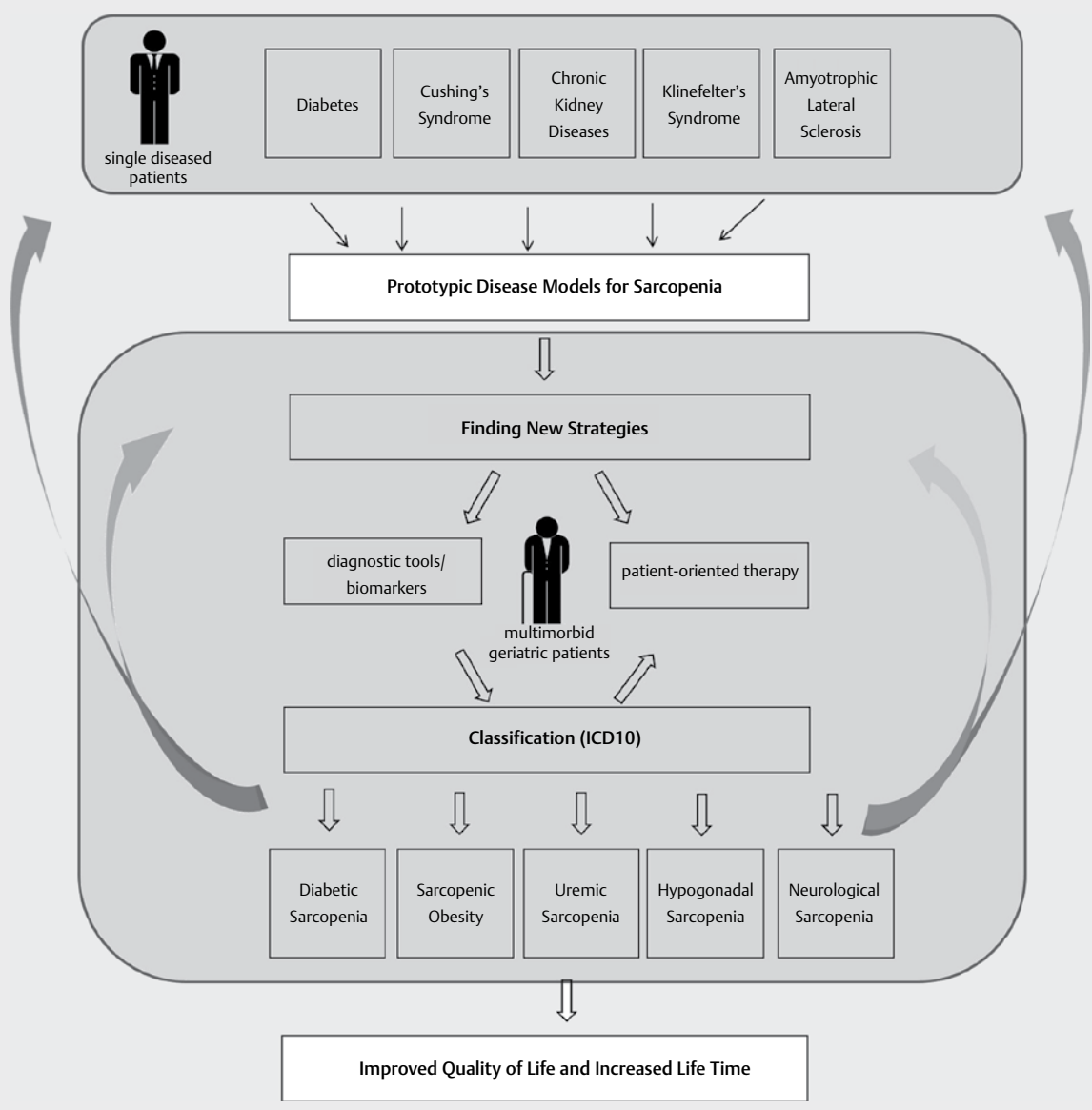

- Fig. 2 Strategy to find new diagnostic tools and a patient-oriented treatment of geriatric sarcopenia by classification. Some diseases (diabetes, Cushing's syndrome (CS), chronic kidney disease (CKD), Klinefelter's syndrome (KS) and amyotrophic lateral sclerosis (ALS)) in non-geriatric patients are known to be associated with muscle degeneration and a so-called "secondary sarcopenia". With the investigation of patients suffering from one of these diseases and thus, being human models for geriatric sarcopenia, we would like to find new strategies to diagnose and treat sarcopenia in geriatric patients. Furthermore, we would like to classify sarcopenic patients into diabetic sarcopenia, sarcopenic obesity, uremic sarcopenia, hypogonadal sarcopenia and neurological sarcopenia to design a patient-oriented treatment strategy. 
in rodent muscle causes hypertrophy and increases glucose disposal, whereas defects in the AKT pathway are detectable in leptin receptor-null mice and obese Zucker rats [14]. A decreased mTOR pathway activity is present in T2D patients [15]. In the insulin resistant state, mTOR signaling is diminished and thus protein synthesis is decreased whereas protein degradation is disinhibited [16]. However, despite the obvious significance of insulin for muscle metabolism and anabolism, its therapeutic potential for the prevention and alleviation of sarcopenia remains unclear. In a retrospective observational study, insulin treatment was protective against the decline of skeletal muscle index (SMI) in the lower, but not the upper extremities [17]. Insulin therapy stimulates protein anabolism in younger, but not in older diabetic subjects and failed to prevent atrophy in elderly [18]. Particularly elderly people may be affected by a differential insulin resistance, in which they might be still sensitive to insulin-mediated glucose uptake, but not anymore to insulin-mediated protein synthesis [19]. In the elderly, supraphysiological insulin doses seem to be necessary to overcome insulin resistance and to stimulate protein synthesis and muscle anabolism [20]. However, permanent hyper-insulinemia has acknowledged negative effects and is therefore an inappropriate approach. Altogether, beneficial effects of insulin on muscle function and mass in elderly are questionable and are certainly no justification for the initiation of an insulin therapy.

It seems encouraging to target insulin resistance itself. The insulin-sensitizing glitazones are promising agents against atrophy [18]. They improve mitochondrial activity and limit protein degradation. In mice, rosiglitazone reduces the activity of caspase- 3 and proteasome in muscle, thus reducing proteolysis and atrophy [21], and treatment with rosiglitazone led to an improvement of muscle mass [14]. The positive effect might be mediated by a disinhibition of the PI3K/AKT pathway via improved insulin sensitivity. Another possible explanation might be the activation of the AKT-mTOR pathway following stimulation of peroxisome proliferator-activated receptor (PPAR) $\mathrm{y}$ by rosiglitazone ( $\triangleright$ Fig. 3 ). However, in elderly obese non-diabetic individuals, pioglitazone did not prevent muscle loss [22] and the unfavorable benefit/risk profile currently limits glitazone use even as anti-diabetic agents.

The biguanide metformin is another drug potentiating insulin actions. Metformin activates AMP-activated protein kinase (AMPK) [23], which is a cellular energy sensor stimulated by a raising AMP/ ATP ratio and increasing glucose and fatty acid uptake and oxidation in skeletal muscle [24]. Thus, AMPK plays an essential role in muscle energy balance during ongoing exercise. Furthermore, AMPK mediates long-term effects of exercise, such as mitochondrial biogenesis [25]. AMPK can furthermore protect against agerelated functional and mitochondrial impairment via promotion of myocyte macroautophagy, which is essential for myocyte maintenance [26]. The beneficial effects of AMPK apply mainly on muscle metabolism. The influence on muscle mass seems to be less favorable. In aging rodents, an inverse relationship of AMPK activation and load-induced muscle hypertrophy was demonstrated [27]. AMPK stimulates myofibrillar protein degradation via increased FOXO expression, down-regulates the mTOR pathway, thus restricting protein synthesis [28], may negatively influence the differentiation of satellite cells [29] and stimulate myostatin expression [30], hereby triggering muscle protein degradation while downregulating muscle protein synthesis ( $>$ Fig. 3 ). An in vitro study showed that metformin increased myostatin expression in cultured myotubes at a concentration of $0.5 \mathrm{mM}$ [31]. Thus, AMPK agonists may have the potential to induce muscle atrophy. The net effect of metformin on muscle function and its clinical impact in alleviating or even aggravating sarcopenia remain to be determined. A case control study indicated a protective effect of metformin against frailty in T2D [32] and a randomized study demonstrated a significantly higher gait speed after a 16-week double blind intervention with

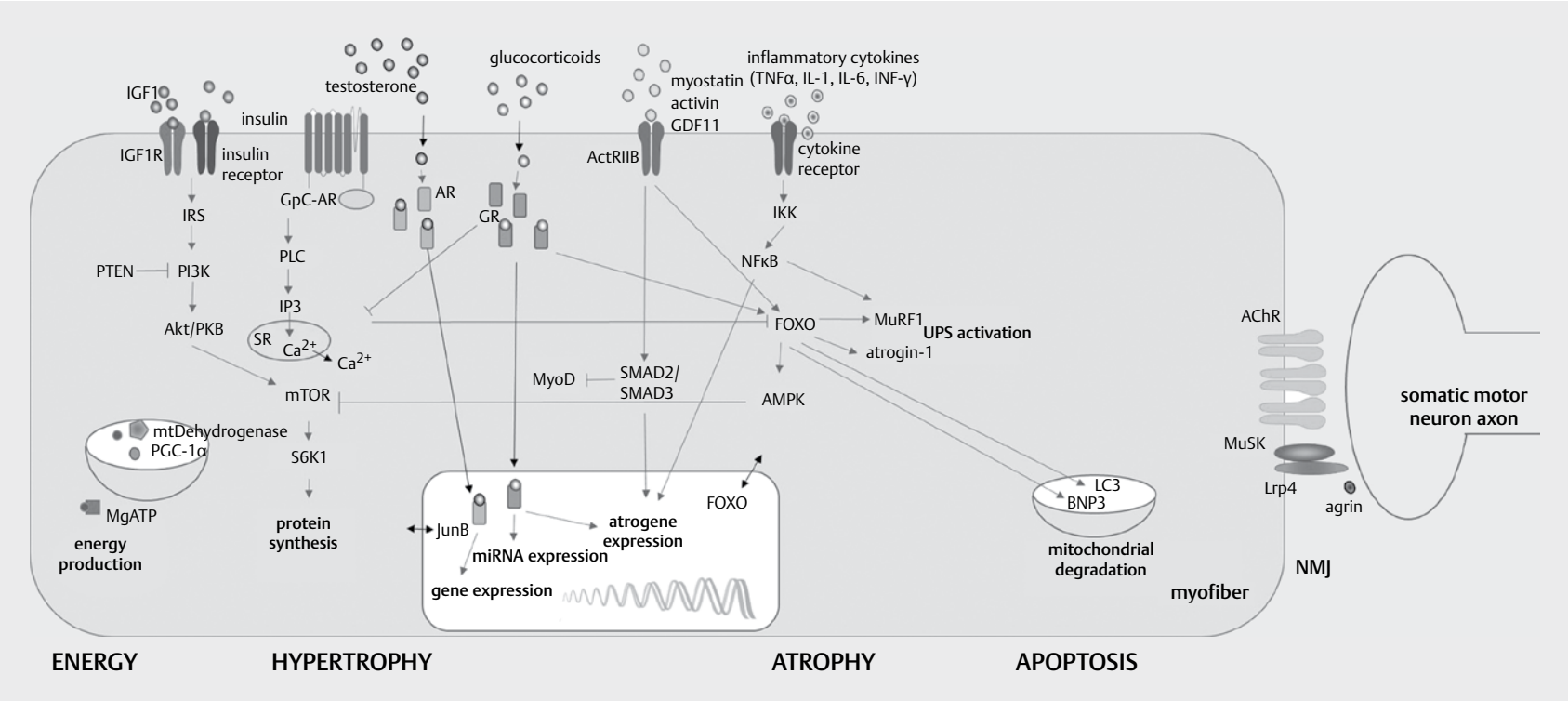

- Fig. 3 Endocrinological and neurological triggered signaling in muscle cells. Hormones can induce various intracellular pathways within myocytes leading to either an increased protein synthesis resulting in hypertrophy or an increase in protein degradation leading to atrophy. Other outcomes of these pathways can be on the positive site an increase in energy production within mitochondria or on the negative site the induction of apoptosis leading to cell death. Main pathways which are involved, are the PI3K-Akt-mTor signaling pathway, AMPK, FOXO or NFKB activation. 
metformin $(3 \times 500 \mathrm{mg} / \mathrm{d})$ in pre-frail elderly participants without diabetes. However, handgrip strength and muscle mass remained unchanged after metformin therapy [33]. These latter results are in line with the above-mentioned AMPK effects. Handgrip strength tests isometric hand muscle contraction, which predominantly involves type II muscle fibers, whose main energy source is anaerobic ATP metabolism. In contrast, the gait speed test requires dynamic rhythmic and repeated muscle contractions, which primarily relay on aerobic glucose metabolism. Altogether, metformin and AMPK activation may alleviate muscle metabolism in endurance exercise, but not muscle mass and/or power.

Blockers of the ATP-sensitive potassium (KATP) channels, such as the sulfonylureas and glinides may induce atrophy. Glibenclamide has been demonstrated to induce muscle atrophy in rats and in humans and repaglinide has been identified as an atrophic agent in preclinical studies [18].

Glucagon-like peptide-1 (GLP-1) exerts anti-apoptotic effects on pancreatic $\beta$-cells and increases glucose sensitivity, proliferation and transcription of proinsulin [34]. The intracellular GLP-1 signaling involves PI3K and other proliferative pathways [18]. Further, GLP-1 has various beneficial extra-pancreatic effects, such as improvement of endothelial and cardiac function and decrease of ischemic myocardial damage [35]. These effects are thought to be at least partially mediated by an improved tissue glucose uptake. In cultured human and rat skeletal muscle cells, GLP-1 increases GLUT4 gene expression and glucose uptake. In rat skeletal muscle, GLP-1 improves insulin sensitivity [36]. But effects of GLP-1 analogues on age-dependent muscle atrophy in animals and humans are currently unknown und warrant further examination. A pilot study involving nine participants revealed a modest increase in skeletal muscle index (SMI) after 24 weeks of liraglutide treatment, whereas body mass index (BMI) and android fat decreased [37].

Dipeptidyl peptidase 4 inhibitors (DPP4i) inhibit endogenous GLP-1 degradation and display a protective effect in preliminary studies. In rats, sitagliptin (40 mg/kg twice daily) restored GLUT4 expression in the heart, soleus und gastrocnemius muscles [38]. In 37 T2D patients treated with DPP4i, the SMI was higher than in T2D patients without DPP4i treatment [39].
Sodium glucose co-transporter inhibitors are also not well studied regarding muscle integrity, but two weeks of dapagliflozin treatment increased insulin-mediated tissue glucose uptake by $18 \%$ [40] and another study found an overall weight loss without reduction of muscle mass in 50 participants with T2D treated with dapagliflozin, indicating a neutral effect of this agent [41].

Taken together, the interwoven pathogenic processes of T2D and sarcopenia may offer chances for shared therapeutic approaches. However, the so far known antidiabetic drug effects on muscle integrity are mainly unfavorable [18] ( is no exercise-mimicking drug and the only established measures to improve both muscle function and insulin sensitivity are exercise-based interventions [42]. Apart from compliance problems, elderly individuals are often too frail to undertake the required degree of exercise. Therefore, currently available data primarily stress the importance of an early detection of muscle deterioration in diabetic patients in order to prevent accelerated progression of both sarcopenia and T2D.

Aside from therapeutic efforts targeting sarcopenia in T2D, it has to be kept in mind that sarcopenia may be one of several major diabetes-related complications that do not necessarily improve with intensification of antidiabetic treatment, but may even be influenced negatively by “overtreatment". Especially in older people, preserving mobility might be more important than a tight blood sugar control. Thus, the current knowledge about the influence of antidiabetic therapy on sarcopenia is - at least to date - one more argument for a less strict and individually adapted antidiabetic therapy in older individuals. Further, whereas reduction of dietary energy intake and weight loss are classically recommended for T2D treatment and improve insulin sensitivity, such interventions may worsen muscle loss in elderly, at least unless concurrent exercise and nutritional supplementation are ensured [43]. Possibly, but unproven, weight loss supported by GLP1-analogues may produce more favorable outcomes.

\section{Cushing's syndrome and sarcopenic obesity}

Cushing's syndrome (CS) characterized by an excessive secretion of cortisol is known to be associated with obesity, muscle atrophy (॰ Fig. 1) and a decline in physical activity. Surgical treatment of

- Table 1 Currently available anti-diabetic drugs and their effects on muscle integrity.

\begin{tabular}{|l|l|l|}
\hline drug class (tested agent) & net effect & mechanisms/present data \\
\hline Insulin & neutral/beneficial & $\begin{array}{l}\text { stimulates protein anabolism (only in younger, not } \\
\text { in older subjects) }\end{array}$ \\
\hline Glitazones (rosiglitazone) & $\begin{array}{l}\text { beneficial, but the risk profile limits } \\
\text { their use }\end{array}$ & $\begin{array}{l}\text { improve mitochondrial activity and decrease } \\
\text { protein degradation; improve muscle mass }\end{array}$ \\
\hline Biguanides (metformin) & unclear & $\begin{array}{l}\text { improvement of muscle metabolism, but potential } \\
\text { to induce atrophy }\end{array}$ \\
\hline Sulfonylurea (glibenclamide) & unfavourable & induce atrophy in rat and human \\
\hline Glinides (repaglinide) & unfavourable & induce atrophy in vitro and in rodents \\
\hline GLP-1 analogues (liraglutide) & possibly beneficial & $\begin{array}{l}\text { increases GLUT4 gene expression and glucose } \\
\text { uptake; increase in SMI }\end{array}$ \\
\hline DPP4-Inhibitors (sitagliptin) & possibly beneficial & higher SMI \\
\hline $\begin{array}{l}\text { Sodium-glucose-co-transporter inhibitors } \\
\text { (dapagliflozin) }\end{array}$ & unclear, possibly neutral & SMI unchanged \\
\hline \multicolumn{2}{|l|}{ Legend: No data are available for Amylin (Pramlintide/Symlin) and glucagon secretion inhibitors. } \\
\hline
\end{tabular}


clinical hypercortisolism due to pituitary adenoma or adrenal gland tumors is effective in the control of cortisol excess, but the functional impairments due to the glucocorticoid-induced myopathy remain despite biochemical remission. Thus, Berr and colleagues could show that hand grip strength and proximal muscle performance were both significantly decreased in patients suffering from active CS compared to obese controls [44]. Six months (short-term) and 2 years (long-term) after surgery, grip strength was still decreased and the chair rising test (CRT) performance also remained impaired. Another study investigating the relationship between CS, muscle atrophy and obesity indicates that CS could even be seen as a prototypic disease model for sarcopenic obesity [45]. CS patients did show a severe decrease in muscle function measured by hand grip strength and CRT, but did not lose muscle mass. Fat infiltration into the muscle might be the reason for decreased muscle quality as CS patients with impaired glucose metabolism showed strongest deterioration of muscle function.

To gain insights into the pathophysiological mechanism leading to muscle atrophy in patients suffering from CS, rodent models were introduced, based on adrenocorticotropic hormone (ACTH) induced hypercortisolism and consecutive muscle atrophy. Thus, rats were infused with $\mathrm{ACTH}$ to produce excessive cortisol in the adrenal glands. Gene expression analysis of skeletal muscle of these rats resulted in an increased expression of $\mathrm{FOXO3a}$ which itself induces the expression of the E3 ubiquitin ligases MuRF1 and atrogin-1 ( Fig. 3). Both of these genes were highly expressed in the infused rats. Furthermore, the FOXO3a promotor was targeted by the activated glucocorticoid receptor (GR) which usually resides in the cytosol, but translocates into the nucleus upon glucocorticoid binding and controls protein degradation in skeletal muscle [46]. A reduction in AKT activity upon GR activation results not only in FOXO3a activation, but also in FOXO1 activation [47]. Both are involved in muscle atrophy through two main pathways: activation of the ATP-UPS and autophagy. The ubiquitination and subsequent degradation of muscle proteins is driven by two E3 ligases MuRF-1 and atrogin-1. MuRF-1 usually targets muscle structural proteins like myosin heavy chain, actin, myosin-binding protein $C$ and troponin I, whereas the targets of atrogin-1 are involved in protein synthesis like eukaryotic translation initiation factor 3 (elF3) and protein translation initiation factor [48]. Besides the UPS activation, FOXO3a also mediates autophagy via the regulation of autophagy-related genes such as microtubule-associated protein $1 \mathrm{~A} / 1 \mathrm{~B}$-light chain 3 (LC3) and BCL2/adenovirus E1B 19-kDa-interacting protein 3 (BNIP3) [49].

As CS is not only associated with muscle atrophy, but in parallel with obesity, a closer look at the molecular pathology of sarcopenia in the context of accompanying obesity seems to be important as obesity appears to be a promoting factor which exacerbates the development of sarcopenia in both, young patients suffering from CS and the older people developing sarcopenia. Obesity is defined as abnormal or extensive fat accumulation that negatively affects health. Sarcopenia and obesity can co-occur, and synergistically are associated with worse functional decline and outcomes than either condition alone [10]. Therefore, Baumgartner and colleagues first described the term sarcopenic obesity as a skeletal mass index that is less than two standard deviations below the sex-specific reference for a young, healthy population, with a percentage of body fat greater than $27 \%$ in men and $38 \%$ in women (roughly a BMI of $27 \mathrm{~kg} / \mathrm{m}^{2}$ ) [50]. Several reasons have been hypothesized how obesity contributes to a decline in skeletal muscle. Under both conditions, in the sarcopenic state as well as in obesity, the muscle is infiltrated with fat, which makes paracrine signaling via (adipo-) cytokines between fat and muscle cells possible. One major cause of muscle loss is the reduced capacity of muscle cell renewal as muscle cell progenitors differentiate due to increased levels of adipokines in an adipocyte-like phenotype. Pro-inflammatory adipokines and cytokines such as interleukin-6 (IL-6), tumor necrosis factor- $\alpha$ (TNF- $\alpha$ ) and C-reaktive protein (CRP) are described as confounders of pathological processes in sarcopenic obesity and lead to a low-grade inflammatory state which will further be described in CKD and uremic sarcopenia. Thus, besides the role of TNF- $\alpha$ in programmed cell death it also upregulates the expression of the muscle atrophying ubiquitin ligase MuRF1 via the nuclear factor 'kappa-light-chain-enhancer' of activated B-cells (NFK-B) pathway. Other causes are the abnormal protein biosynthesis rate and anabolic resistance to exercise. There seems to be a cross-talk between the hypothalamo-pituitary axis and the nutritional status which leads to a higher catabolic state of metabolism in obese patients and a higher susceptibility of muscle wasting under energy restriction [51]. Further mechanisms are the obesity derived intracellular lipotoxicity which leads to an increase of lipids and fatty acids in muscle cells causing oxidative stress. Reactive oxygen species (ROS), chronic inflammation, insulin resistance, increased levels of leptin and a decrease of adiponectin lead to mitochondrial damage and apoptosis in skeletal muscle. Further, pituitary induced high circulating levels of glucocorticoids in CS may directly modulate bone remodeling and metabolism causing deterioration of the structural integrity of bones which is associated with a high risk of fractures [52]. Thus, CS is often accompanied by a secondary osteoporosis which leads in combination with muscle atrophy and obesity even to an osteosarcopenic obesity, a new phenotype in geriatrics [53]. Hypercortisolism affects bone metabolism with various mechanisms. It is known that glucocorticoid excess inhibits bone formation, reduces the replication of bone forming cells, prevents the differentiation of osteoblasts, induces apoptosis of osteoblasts by activating caspase 3 and inhibits collagen type I synthesis [54]. Treatment of pituitary hormone excess such as surgery of pituitary adenomas improve skeletal health in some patients, whereas others seem to remain a high risk of fractures. As muscle, fat and bone tissue exert an immense cross-talk, it would be interesting to study patients suffering from CS and associated secondary osteosarcopenic obesity in more detail to find out more about pathologies leading to geriatric sarcopenia often accompanied by osteoporosis.

Since about three out of four European adults are overweight, and sarcopenia and obesity can co-occur or even both occur in patients suffering from CS, a closer look at the molecular pathology of the CS driven sarcopenia/sarcopenic obesity is important [55]. The profound decrease in life quality of patients suffering from CS with muscle function impairments emphasize the need for further studies clarifying the exact mechanisms by which muscle function is affected. However, this knowledge about potential pathomechanisms in steroid-driven myopathy could also be beneficial for elder 
sarcopenic obese patients as research in these patients is hampered by confounding comorbidities and polypharmacy. As CS patients are frequently free of comorbidities and as CS is potentially curable, $\mathrm{CS}$ could be a prototypic disease model for further research in sarcopenic obesity.

Pituitary induced high circulating levels of glucocorticoids in CS may directly modulate bone remodeling and metabolism causing deterioration of the structural integrity of bones which is associated with a high risk of fractures [52]. Thus, CS is often accompanied by a secondary osteoporosis which leads in combination with muscle atrophy and obesity even to an osteosarcopenic obesity. Hypercortisolism affects bone metabolism with various mechanisms. It is known that glucocorticoid excess inhibits bone formation, reduces the replication of bone forming cells, prevents the differentiation of osteoblasts, induces apoptosis of osteoblasts by activating caspase 3 and inhibits collagen type I synthesis [54]. Treatment of pituitary hormone excess such as surgery of pituitary adenomas improve skeletal health in some patients, whereas others seem to remain a high risk of fractures. As muscle, fat and bone tissue exert an immense cross-talk, it would be interesting to study patients suffering from CS and associated secondary osteosarcopenic obesity in more detail to find out more about pathologies leading to geriatric sarcopenia often accompanied by osteoporosis.

\section{Chronic kidney disease and uremic sarcopenia}

Uremic sarcopenia describes prevalently occurring muscle abnormalities developing in the uremic milieu in patients suffering from chronic kidney disease (CKD). As the loss of muscle mass in uremic patients is much more intensive and the first signs of sarcopenia are observed in younger patients than usually expected, the pathogenic mechanisms involved might give important insights when prototypically modelling sarcopenia in human patients. However, muscle abnormalities in renal patients receiving dialysis are usually not defined by a change in muscle physiology, but instead by reduced muscle force, significant muscle wasting and selective structural changes including type II fiber atrophy (with a greater fiber atrophy of type IIB compared to type IIA), small cross-sectional area (CSA), fiber grouping and mitochondrial aberrations with a decrease in mitochondrial enzyme concentration and activity [56]. The prominent and progressive reduction of muscle mass in uremic sarcopenia appears to be the answer of a disrupted skeletal muscle homeostasis with a multifactorial etiology involving hormonal, immunologic and myocellular causes, inflammation, reduction of protein intake, metabolic acidosis, increased angiotensin II, abnormalities in insulin/insulin-like growth factor 1 (IGF-1), myostatin expression, satellite cell inactivation, mechanical changes like physical inactivity and comorbidities [56].

Many of these numerous causes leading to uremic sarcopenia have an increase in muscle proteolysis and a decrease in muscle protein synthesis in common. The major cause in CKD muscle wasting is thought to be the activation of the UPS which leads to excessive protein degradation and especially an increase in the cleavage of the $14 \mathrm{kDa}$ actin fragment [57]. Both, inflammation and metabolic acidosis strongly trigger the activation of the ATP-UPS. Lowgrade inflammation which is common in CKD is marked by increased circulating levels of the inflammatory markers CRP, IL-6 and TNF- $\alpha$. Thus, it was shown, that muscle mass in dialysis patients inversely correlates with an increase in CRP and IL-6 levels. However, TNF- $\alpha$ as the major inflammation factor that triggers protein degradation, directly activates the ubiquitin proteasome and induces muscle wasting through the activation of the NFKB pathway by increasing the expression of antrogenes (MuRF-1, atrogin-1 and MAFbx) or by attenuation of the insulin-stimulated protein synthesis via FOXO [58].

Metabolic acidosis as another major causative pathological mechanism of sarcopenia development in CKD, occurs mainly through a strong accumulation of the uremic toxin indoxyl sulfate (IS), which activates on the one hand also the UPS, but also leads on the other hand to oxidative stress and metabolic changes in muscle cells. IS moreover induced mitochondrial network disintegration as consequence to metabolic changes such as upregulation of antioxidative responses (pentose phosphate pathway and glutathione metabolism) which were related to nuclear factor-2 (Nrf2) activation, and impaired mitochondrial ATP production due to a downregulation of the tricyclic acid (TCA) cycle, the glutamine metabolism and the mitochondrial oxidative phosphorylation. The IS induced mitotoxicity and immense ATP shortage is especially detrimental in skeletal muscle which are highly metabolic and require vast quantities of mitochondria for ATP production. However, the association between plasma IS levels and muscle mass was also clinically examined in CKD patients undergoing peritoneal dialysis and indicates a decrease in muscle mass when IS levels are elevated [59]. Besides the above hypothesized pathomechanistic pathway induced by increased IS levels, other studies describe a close relationship between IS accumulation, oxidative stress, inflammation and muscle atrophy. Thus, accelerated IS causes muscle breakdown not only through an increase of ROS, but also through an increase of inflammatory cytokines such as TNF- $\alpha$, IL- 6 and transforming growth factor- $\beta 1$ (TGF- $\beta 1$ ) resulting in an enhanced expression of the muscle atrophy related genes myostatin and atrogin-1.

The renin-angiotensin-aldosterone-system (RAAS) is highly activated under conditions of CKD and also seems to provide a strong input to the development of uremic sarcopenia. Thus, angiotensin II is highly elevated, which leads to a decrease of phospho-actin, resulting in an increase of caspase- 3 which enhances actin cleavage. Not only muscle proteolysis is elevated, but also apoptosis of muscle cells, both promoting muscle wasting. Angiotensin II is also responsible for low circulating and skeletal IGF-1 levels which enhances muscle wasting as well [60]. Other chronic conditions which are associated with a dysregulation of RAAS and muscle dystrophy are congestive heart failure and liver cirrhosis [61,62].

Another risk factor for the development of sarcopenia in CKD represents the disturbances in insulin levels and resistance, which is described to be low in CKD. One pathway through which insulin is involved in uremic myopathy is the activation of the UPS through $\mathrm{PI} 3 \mathrm{~K}$ activation. But PI3K can also activate an intracellular signaling cascade involving AKT-P, which is involved in protein degradation through FOXO and in decreased protein synthesis through GSK-1 and mTOR ( $>$ Fig. 3 ). However, low insulin resistance, which correlates linearly with the decline of renal function, leads also to a dramatic decrease in the use of glucose as energy source and an impaired glucose metabolism [63]. 
Other mechanisms that might influence protein homeostasis are disturbances in appetite with changes in ghrelin and leptin levels and also the inactivation of satellite cells which then reduce their MyoD and myogenin expression resulting in a reduced replacement of injured muscle cells [64].

Uremic muscle wasting is also highly complex and progressive when comparing with sarcopenia. The pathogenesis of both, geriatric and uremic sarcopenia is similar, which makes it important to investigate the commonalities in an interdisciplinary approach.

\section{Klinefelter's syndrome and hypogonadal sarcopenia}

Klinefelter's syndrome (KS) (47XXY) as the most abundant sex chromosome disorder is characterized by hypogonadism, gynecomastia and azoospermia. It is strongly associated with a changed body composition, leading to an increase in fat mass and a decrease in both, muscle mass and bone mineral mass [65]. Affected men show also lower aerobic capacity and a diminished muscle strength and performance. Furthermore, KS often goes along or even results in T2D, metabolic syndrome and cardiovascular events [66]. The treatment of choice is the supplementation with testosterone which is the most widely prescribed medication in the USA so far. As with advancing age hypogonadism appears in elderly people leading to a decrease of muscle mass and performance, KS seems to be a good prototypic disease model to study hypogonadism and decreased levels of testosterone in a young population leading to the induction of muscle wasting and thus, a "secondary sarcopenia" that we name hypogonadal sarcopenia [67].

Testosterone as the most important anabolic steroid hormone is known to be involved in a variety of developmental and biological processes including the maintenance of muscle and bone and the inhibition of adipogenesis. With advancing age, the muscle mass, which is in the age of 40 years about $60 \%$ of total body mass, decreases up to about $40 \%$ when reaching the age of 70 years $[67,68]$. At the same time, bone mineral density decreases and adipose tissue increases leading to sarcopenia, frailty and obesity. Testosterone levels decrease dramatically with age and age-induced hypogonadism is not rare in today's aging population. Concerning the decline of testosterone levels, Feldman et al. preformed some cross-sectional and longitudinal studies [69]. They could determine in a first study that total testosterone levels decline $0.8 \%$ per year. Both, free and albumin-bound testosterone decrease $2 \%$ per year after the $4^{\text {th }}$ decade of life. In a second study, which was longitudinal, the total testosterone decline was $1.6 \%$ per year and the decline of bioavailable testosterone was $2-3 \%$ per year. In a third study they could show that the testosterone level in general and the free testosterone index declined progressively from the $3^{\text {rd }}$ to $9^{\text {th }}$ decade of life with a decline for total testosterone levels being $0.11 \mathrm{nmol} / \mathrm{L}$ per year and being $0.0049 \mathrm{nmol}$ testosterone per nmol sexual hormone binding globuline (SHBG) per year for the free testosterone index. Several studies followed from other groups leading to the same results that testosterone levels decrease with age [69].

The reason for the negative effects induced by low testosterone levels while aging, is not only a low androgen production in general, but also an accelerated testosterone metabolism or malfunctioning androgen receptors. Testosterone seems to build an extrinsic factor which prevents sarcopenia or will enhance when levels are low. The exact metabolic function of testosterone in promoting skeletal muscle function remains poorly understood. It is known or seems to be important, that testosterone increases muscle protein synthesis, which is important for muscle formation, growth and maintenance. Furthermore, testosterone plays also a crucial role in muscle regeneration via the activation of satellite cells [67].

Many studies have been performed to investigate the effects of testosterone, but also to find out more about the exact role of testosterone in muscle, bone and fat metabolism. The testosterone replacement therapy (TRT) is widely used [67]. However, the sample sizes of the studies are often small. Furthermore, most of the studies were performed in the USA and Europe, which does not allow any prediction about i. e. the Asian population. Results are very contradictory. Several studies showed an association of frailty and testosterone concentrations, clearly indicating that testosterone plays an important role in the development of sarcopenia [70]. Muscle mass and muscle strength could be shown to be related with testosterone concentrations in many studies. Interestingly, testosterone level and physical performance showed rather inconsistent relationships. Most likely this can be explained by a diversity of used tests. Physical performance is difficult to measure in the elderly and depends on many factors in addition to muscle strength. Therefore, testosterone supplementation was given to young and middle-aged men suffering from hypogonadism and indeed, beneficial effects could be determined like the increase in weight and muscle mass, as well as a reduction in fat mass, whereas the side effects were very low [69]. However, the clinical advantages of a TRT in elderly men with age-associated decline of testosterone remains unclear. Thus, since the risk/benefit ratio of androgen replacement therapy is unclear, it is suggested to avoid this therapy in elder people until better outcome data are available. Although testosterone is used since 1940 as therapeutic drug, the negative side effects especially cardiovascular events are redoubtable. Therefore, SARMs (selective androgen receptor molecules) were developed which will be discussed later in the part of managing and treating sarcopenia [71].

Therefore, patients suffering from KS seem to represent a good prototypic disease model to investigate in both, young (40 years) and very young (20 year) men the tremendous effects of hypogonadism and to study the outcome of testosterone supplementation on intracellular signaling and muscle mass as well as muscle performance.

\section{Amyotrophic lateral sclerosis and neurogenic sarcopenia}

Amyotrophic lateral sclerosis (ALS) is a rapid progressive neurodegenerative disease caused by degeneration of brachial and lumbar somatic motor neurons resulting in spinal, bulbar, respiratory and axial muscle weakness. Furthermore, the neuromuscular junction degenerates and the skeletal muscle becomes denervated thus causing initial muscles weakness and following atrophy. Reported age of onset for ALS varies between 40 and 65 years [72]. Mean survival is typically between 3 and 5 years after disease onset. Respiratory failure occurs during disease progression, which is the main cause of death in ALS [73]. Today, most cases are considered as a sporadic type (sALS), however there is growing evidence for ALS 
associated gene mutations [74]. There is clinical and animal experimental debate that pathological changes might occur at the neuromuscular junction (NMJ) prior to motor neuron degeneration and onset of clinical symptoms [75]. This hypothesis proposes that ALS is a distal axonopathy in the early phase of the disease onset [76]. The "dying-back" or "transsynaptic degeneration" theory has obtained much attention in recent years in the context of ALS pathophysiology. According to this hypothesis, motor neurons and nerve terminals show pathological changes prior to motor neuron degeneration and the onset of clinical symptoms. Interestingly, evidence of distal axonopathy has been found in other neurodegenerative diseases like Alzheimer's disease or Parkinson's disease, where axonal defects occur prior to cell death and the loss of axonal function correlates strongly with the onset of functional decline $[77,78]$. These two neurodegenerative mechanisms, loss of motor neurons and degeneration of NMJs, are also found as causes for sarcopenia. While disease progression in ALS is rapid and leads through muscular respiratory failure to death, neurogenic sarcopenia is a chronic condition leading to dysmobility, falls, fractures and loss of independency at advanced age in the ninth decade.

Irrespectively of the pathomechanisms, both diseases are characterized by a loss of motor units and muscle fiber atrophy. A motor unit comprises a motor neuron and the group of muscle fibers it innervates. The number of muscle fibers innervated by a motor neuron (size of motor unit) varies widely from very small innervation ratios in the hand and eye muscles ( $<10$ fibers per motor neuron) to very large innervation ratios in the trunk and proximal limb muscles (> 500 fibers per motor neuron). Several electrophysiological Motor Unit Number Estimation (MUNE) methods for estimating the number of motor units have been used in ALS as an objective measurement to monitor disease progression [79]. The MUNE technique for human muscle was described for the first time in 1971 [80]. This technique has been developed further and modified in different ways over the years. Many MUNE techniques are time-consuming and technically difficult to perform or invasive with discomfort for the patients. For this reason, Nandedkar et al. introduced a technique called the Motor Unit Number Index (MUNIX) in 2004 to obtain a parameter related to the number and size (MUSIX) of motor units (MU) [81]. This technique is non-invasive, and easy and quick to perform. The Motor Unit Number Index is amethod for assessing the number (MUNIX) and size (Motor Unit Size Index - MUSIX) of motor units. Investigations have demonstrated the good reliability and validity of MUNIX values $[82,83]$. Investigations to study the relevance of MUNIX in sarcopenic patients have shown that MUNIX values of sarcopenic patients lie between healthy subjects and ALS patients [84]. This finding indicates that loss of motor neurons most probably plays a prominent pathogenic role in the development of sarcopenia. In another study it was shown that patients with pathological MUNIX and MUSIX have a significant increased odds ratio of 3.09 of being sarcopenic [85].

Compared to this neurocentric view of muscle fiber atrophy due to a transsynaptic degeneration of the motor neuron, the primary degeneration of the NMJ and its dying-back mechanism constitute a myocentric view of muscle fiber loss. In this context the motor neuron synthetized proteoglycan agrin, which is secreted in the synaptic cleft and induces acetylcholine receptor (AChR) clustering in the postsynaptic membrane, is important for the formation and stability of the NMJ [86]. The postsynaptic induced effect of agrin is mediated through activation of the muscle specific kinase (MuSK) and low-density lipoprotein receptor-related protein (Lrp4) in the plasma membrane. Agrin is inactivated by cleavage from neurotrypsin, a synaptic protease, which frees a soluble $22 \mathrm{kDa}$ C-terminal agrin fragment (CAF) that can be detected in human serum [87]. Experiments with transgenic mice overexpressing neurotrypsin in spinal motor neurons have shown the full sarcopenia phenotype, including a reduced number of muscle fibers, increased heterogeneity of fiber thickness, more centralized nuclei, fiber-type grouping and an increased proportion of type I fibers. Several studies have shown a correlation between CAF concentrations and muscle mass and or muscle performance, qualifying CAF as a marker for sarcopenia [88]. Interestingly, the injection of a soluble neural agrin fragment has considerably improved muscle pathology caused by the disassembly of the NM] in mice [89].

The described overlap in the pathogenesis of both diseases makes it obvious to study ALS, especially in early stages of the disease, in parallel with neurogenic sarcopenia. This interdisciplinary approach between myologists, neurologists and geriatricians may lead to new translational diagnostic and therapeutic steps in the treatment of both diseases.

\section{Potential Biomarkers for Sarcopenia}

\section{Techniques}

- Table 2 represents an overview of the current knowledge about diagnostic tools and biomarkers which play a role in the detection of sarcopenia risk, development and process. Some of these biomarkers are also involved to a greater extent in some of the above described prototypic disease models for sarcopenia. However, up to now, the clinical gold standard to diagnose the decline in muscle mass is dual-energy X-ray absorptiometry (DEXA). For research, computed tomography $(\mathrm{CT})$ and magnetic resonance tomography (MRT) are also used [90]. Limitations of these techniques are not only, that they are quite expensive and only available in large clinics and institutions, but also that they are incapable to detect the risk of developing a muscle atrophy when the muscle is not wasted yet [91].

For muscle function and strength measurements a variety of procedures exist. For geriatric patients hand grip strength and gait speed are the gold standards. However, they are always dependent on motivation and cooperation of patients in all age groups and time consuming in clinical practice. Diagnostic tools discovered in the research of ALS pathology which are also used in (neurogenic) sarcopenia diagnosis are the estimation of the motor unit number and size by MUNIX and MUSIX [81].

Thus, new biomarkers and diagnostic tools, which are cheap and easily available are tremendously needed. Regarding the knowledge about the sarcopenic pathophysiology and the pathophysiology of the prototypic disease models of sarcopenia in non-geriatric patients with diseases associated with "secondary sarcopenia" or muscle wasting, promises many potential biomarkers. These markers need to be investigated in more detail ( $>$ Table 2 ). 
- Table 2 Overview about diagnostic tools and biomarkers for sarcopenia.

\begin{tabular}{|c|c|c|}
\hline diagnostic tools/biomarkers for sarcopenia & additional information & diseases \\
\hline \multicolumn{3}{|l|}{ techniques: } \\
\hline $\begin{array}{l}\text { Computed Tomography (CT), Magnetic Resonance Tomography } \\
\text { (MRT), Dual-Energy X-ray Absorptiometry (DEXA }\end{array}$ & measurement of muscle mass & all \\
\hline $\begin{array}{l}\text { Motor Unit Number Estimation (MUNE), Motor Unit Number } \\
\text { Index (MUNIX), Motor Unit Size Index (MUSIX) }\end{array}$ & $\begin{array}{l}\text { elecrophyiological method for motor unit number and size } \\
\text { estimations }\end{array}$ & ALS \\
\hline \multicolumn{3}{|l|}{ histological aspects: } \\
\hline muscle fiber number, fiber grouping, fiber size heterogeneity & $\begin{array}{l}\text { decrease in atrophying muscle resulting in reduced muscle } \\
\text { mass, strength and power }\end{array}$ & ALS; CKD \\
\hline $\begin{array}{l}\text { motor neuron degeneration/aonopathy/loss of neuromuscular } \\
\text { junction (NMJ) }\end{array}$ & $\begin{array}{l}\text { motor neurons and nerve terminals show pathological changes } \\
\text { prior to motor neuron loss }\end{array}$ & ALS \\
\hline \multicolumn{3}{|l|}{ type II muscle fiber atrophy } \\
\hline cross-sectional area (CSA) of fibers & CSA smaller in atrophying muscle & ALS; CKD \\
\hline $\begin{array}{l}\text { giant and non-functional or old mitochondria often with no } \\
\text { outer membrane }\end{array}$ & occur in atrophying muscle & CKD \\
\hline \multicolumn{3}{|l|}{ blood and/or urinary markers: } \\
\hline myostatin & inhibitor of muscle growth & Diabetes \\
\hline follistatin (FST) & myostatin inhibitor & Diabetes; CS \\
\hline creatinine & $\begin{array}{l}\text { urinary creatinine levels are often elevated when muscle mass } \\
\text { cheanges }\end{array}$ & CKD \\
\hline C-terminal agrin fragments (CAF) & $\begin{array}{l}\text { responds to resistance exercise training; involved in motor } \\
\text { neuron loss. }\end{array}$ & ALS \\
\hline ciliary neuotrophic factor (CNTF) & decreased levels result in lower hand grip strength & ALS \\
\hline indoxyl sulfate & causes metabolic acidosis and often leads to muscle atrophy & CKD \\
\hline angiotensin II & $\begin{array}{l}\text { increased levels lead to enhanced muscle proteolysis and an } \\
\text { increased muscle cell apoptosis }\end{array}$ & CKD \\
\hline \multicolumn{3}{|l|}{ signaling molecules: } \\
\hline $\begin{array}{l}\text { muscle.specific RING-finger } 1 \text { (MURF1 = TRIM63), ubiquitin } \\
\text { tripartite motif containing protein } 32 \text { (TRIM32) and atrogin } 1\end{array}$ & atrogene & CS, Diabetes \\
\hline insulin growth factor-1 (IGF-1) & increase food intake and growth hormone & CS, CKD \\
\hline \multicolumn{3}{|l|}{ inflammatory markers: } \\
\hline interleukine-1 (IL-1) & increased inflammatory signalsare involved in muscle atrophy & CKD \\
\hline $\begin{array}{l}\text { interleukine-6 (IL-6), tumor necrosis factor } \alpha \text { (TNF- } \alpha) \text {, } \\
\text { C-reactive protein (CRP) }\end{array}$ & increased inflammatory signalsare involved in muscle atrophy & Diabetes, CKD \\
\hline \multicolumn{3}{|l|}{ tartrate- resistant acid phosphatase 5a (TRACP5a) } \\
\hline \multicolumn{3}{|l|}{ hormones: } \\
\hline testosterone & decline in muscle mass and strength & KS \\
\hline insulin & insulin resistance often leads to muscle degeneration & $\begin{array}{l}\text { Diabetes, CKD, } \\
\text { CS }\end{array}$ \\
\hline \multicolumn{3}{|l|}{ microRNAs: } \\
\hline miR-1, miR-208, miR-486 & $\begin{array}{l}\text { results in decrease of sattelite cell renewal and decreased } \\
\text { IGF1-PI3K-Akt signaling }\end{array}$ & CS \\
\hline $\begin{array}{l}\text { Legend: There are improving techniques and different biomarke } \\
\text { investigation, but are not used in a standardized diagnosis today } \\
\text { previous described model diseases which often lead to secondar } \\
\text { diseases; CS: Cushing's syndrome.) }\end{array}$ & $\begin{array}{l}\text { determine sarcopenia risk, development and process. Most of } \\
\text { ne of these biomarkers seem to be involved to a greater exten } \\
\text { copenia. (Abbreviations: ALS: amyotrophic lateral sclerosis; CK }\end{array}$ & $\begin{array}{l}\text { m are under } \\
\text { some of the } \\
\text { hronic kidney }\end{array}$ \\
\hline
\end{tabular}

\section{Histological aspects}

In general, all muscle wasting conditions show enormous histological abnormalities including one or more of the following described effects: reduced muscle fiber number, fiber type grouping, heterogeneity in fiber size, giant and non-functional mitochondria as well as old mitochondria without outer membrane. Many of these are seen especially in the pathology of CKD resulting in uremic sarcopenia. Other histological changes are motor neuron degeneration, axonopathy and loss of NMJs which is of course one main aspect in ALS pathology leading to neurogenic sarcopenia ( $\triangleright$ Table 2 ).

\section{Blood and/or urinary markers}

In sarcopenic patients numerous proteins and molecules which are dysregulated can be detected in increased or decreased levels often even in the blood serum or plasma and in urine samples. Therefore, 
many are suggested as potential biomarkers and can be used as diagnostic tools for sarcopenia ( $>$ Table 2 ). In degenerating muscle, myostatin as the main autocrine inhibitor of muscle growth is most probably highly upregulated and represents a putative marker for muscle atrophy, which is highly relevant in diabetic sarcopenia, but also in all other types of "secondary sarcopenia" [92]. In line, follistatin (FST) as naturally occurring strong inhibitor of myostatin signaling and thus, being a positive regulator of muscle growth, is also suggested as a potential biomarker. Urinary creatinine levels are often elevated, when muscle mass is changed in CKD. Another circulating biomarker is CAF which is increased in $1 / 3$ of sarcopenic patients and represents an evidence for motor neuron loss. It is decreased in sarcopenia, but responds positively to resistance exercise training [93]. On the other side, factors like ciliary neurotrophic factor (CNTF), which is also known to play a crucial role in ALS pathology, are upregulated in sarcopenia shown by a lowered hand grip strength [85]. Moreover, mitochondrial enzymes are suggested as indicators for sarcopenia. Thus, levels of peroxisome proliferator-activated receptor c coactivator $1 \alpha$ (PGC- $1 \alpha)$ decreases dramatically in sarcopenia [94]. It is important for mitochondrial function and integrity, decreases FOXO and MURF-1 signaling and its decrease results in an increase of TNF- $\alpha$ [95]. The cross-talk of mitochondrial enzymes with cytoplasmic or nuclear molecules is disturbed and there are also a lot of signaling molecules which can serve as biomarkers in atrophying muscle conditions. Changed levels are often detected in CKD and uremic sarcopenia ( $\triangleright$ Table 2).

\section{Signaling molecules}

Atrogenes like the muscle-specific RING finger 1 (MURF1 = TRIM63) and ubiquitin tripartite motif containing protein 32 (TRIM32) are upregulated as well as atrogin-1 (

\section{Inflammatory markers}

Muscle degradation goes also along with an increase of inflammatory markers and cytokines such as IL-1, IL-6, TNF- $\alpha$ and CRP. Chronic inflammation and thus a change in inflammatory factors is often seen in pathologies of endocrine diseases such as CS, diabetes and CKD ( Table 2).

\section{Hormones}

Also hormonal factors have been postulated to be good biomarkers as they are substantially involved in sarcopenia development. Testosterone levels decrease about $1 \%$ per year from the age of 35 years. This decline is followed by a reduction in muscle mass and strength and plays of course the most significant role in hypogonadism [96]. Insulin resistance is also often associated with atrophying muscle and is involved not only in diabetes, but also in CS and CKD development ( $\triangleright$ Table 2 ).

\section{microRNAs}

Besides all these proteins as biomarkers, also molecules like miRNAs demonstrate promising markers for sarcopenia and muscle degeneration. The decreased levels of miR-1 (often associated with cortisol excess and thus relevant for (S), miR-208 and miR-486 result in a decrease of satellite cell renewal and decreased IGF1-PI3KAKT signaling leading to muscle degeneration [6] (> Table 2).

\section{Potential Therapeutics and Future Classification}

\section{General}

Up to now, no pharmacological treatment for sarcopenia is available, although a lot of substances are currently in clinical studies ( Table 3). Today's recommendation in general treatment against sarcopenia is: (I) resistance training, which decreases especially the functional decline in lower limb muscles, (II) together with a high protein diet, in particular leucine-rich protein (whey) to enhance muscle mass and function, (III) often in combination with vitamin D supplementation to increase muscle strength $[6,7]$ (

\section{Testosterone and selective androgen receptor modulators}

The first therapeutic agent, that was used against muscle wasting was testosterone in the early 1940ies [97]. Testosterone levels physiologically decrease about $1-2 \%$ per year in both, men and woman, starting in the mid-thirties [68]. A lot of focus was put on testosterone as it seems to be an all-round hormone in regard to muscle mass, strength and power. It seems to be important in satellite cell activation and increased muscle protein synthesis ( Fig. 3) [6, 98]. It is even possible to decrease the amount of fat mass by a considerable reduction of adipose-derived stem cells and to increase bone strength and bone mineral density [70]. However, a lot of studies using testosterone as treatment of muscle wasting were carried out and besides all the positive effects, unfortunately also a lot of negative side effects were occurring such as cardiovascular events which led to a new way of treatment by using selective androgen receptor modulators (SARMs). One of the first SARMs used is nadrolone, a steroidal SARM, which was proved to increase muscle fiber area and thus, muscle mass, but there was no increase in muscle strength [6]. Another steroidal SARM is known as MK0773 which also increases muscle mass, but the study was earlier terminated because of increased signals of heart failure [6]. Two non-steroidal SARMs are LGD-033 and BMS-564929. Although a further SARM called enobosarm could maintain body mass and increase stair climb power in patients with cancer, SARMs have shown no advantage over testosterone why the way in developing a treatment against muscle wasting went back to testosterone and its new trials [99] (॰ Table 3).

\section{Ghrelin and growth hormone}

Ghrelin, ghrelin agonists (anamorelin, BIM-28125, BIM-28131 and macimorelin) or ghrelin receptor agonists (capromorelin and MK0677) were shown to be beneficial. Ghrelin is important for an increase in food intake, increased levels of growth hormone $(\mathrm{GH})$ and an increase in muscle mass. However, an increase in muscle strength was missing. Using directly $\mathrm{GH}$ was another attempt to treat muscle wasting conditions. Although GH treatment showed in old men a significant increase in lean body mass, there were many associated side effects such as arthralgia, muscle pain, edema, carpal tunnel syndrome and hyperglycemia [6] (॰ Table 3). 
- Table 3 Overview about current treatment strategies for sarcopenia.

\begin{tabular}{|c|c|c|}
\hline Therapy/drug & Effects & Side effects \\
\hline resistance exercise & $\begin{array}{l}\text { increase muscle mass and power; } \\
\text { reversing frailty }\end{array}$ & potential for falls; muscle injuries \\
\hline whey protein & $\begin{array}{l}\text { increase of muscle mass; synergy } \\
\text { with exercise to increase muscle } \\
\text { strength and power }\end{array}$ & minimum increased creatine levels \\
\hline vitamin D & enhances muscle strength & - \\
\hline testosterone & $\begin{array}{l}\text { increase muscle mass, strength, } \\
\text { power and function }\end{array}$ & $\begin{array}{l}\text { potential for falls; muscle injuries; minimal increased } \\
\text { creatinine levels; fluid retention; increased hematocrit; } \\
\text { short term worsening of sleep apnea; effects on prostate } \\
\text { cancer; possible increase in cardiovascular events }\end{array}$ \\
\hline $\begin{array}{l}\text { SARMs } \\
\text { Nadrolone (steroidal SARM); MK0773 } \\
\text { (TFM-4AS-1) (4-aza steroidal SARM); LGD-4033, } \\
\text { BMS-564929 (non-steroidal SARM); Enobosarm }\end{array}$ & $\begin{array}{l}\text { increases muscle mass, fiber area, } \\
\text { small increase in muscle power }\end{array}$ & increased cardiovascular failure \\
\hline $\begin{array}{l}\text { ghrelin } \\
\text { Anamorelin, BIM-28125, BIM-28131, macimor- } \\
\text { elin (agonist); Capromorelin, MK-0677 } \\
\text { (receptor agonist) }\end{array}$ & $\begin{array}{l}\text { increases muscle mass, strength and } \\
\text { appetite, reduces TNF- } \alpha \text { and } \\
\text { myostatin levels }\end{array}$ & Fatigue; atrial fibrillation; dyspnea increased heart failure \\
\hline growth hormone & $\begin{array}{l}\text { increases nitrogen retention; } \\
\text { increases muscle mass, but not } \\
\text { strength }\end{array}$ & $\begin{array}{l}\text { Arthralgia; muscle pain; edema; carpal tunnel syndrome; } \\
\text { hyperglycemia }\end{array}$ \\
\hline $\begin{array}{l}\text { myostatin and activin II receptor inhibitors } \\
\text { MYO-029, AMG 745, LY2495655, REGN1033 } \\
\text { (myostatin antibody); ACE-011, ACE-083 } \\
\text { (Activinll receptor ligand trap); Bimagrumab } \\
\text { (Activin receptor inhibitor) }\end{array}$ & $\begin{array}{l}\text { enhances muscle mass; increases } \\
\text { muscle fiber diameter; decreases fat } \\
\text { mass; increases bone mass }\end{array}$ & $\begin{array}{l}\text { urticaria; aseptic meningitis; telangiectasia, epistaxis, and } \\
\text { changes in gonadotrophin levels }\end{array}$ \\
\hline $\begin{array}{l}\text { mixed agonist/antagonist B1, B2, B3 activity } \\
\text { Espindolol }\end{array}$ & $\begin{array}{l}\text { increases muscle mass, decreases fat } \\
\text { mass, increased hand grip strength }\end{array}$ & \\
\hline $\begin{array}{l}\text { angiotensin converting enzyme inhibitor } \\
\text { Perindopril }\end{array}$ & $\begin{array}{l}\text { increases distance walked, improves } \\
6 \text {-min walking distance, decreases } \\
\text { hip fractures }\end{array}$ & Hypotension; hyperkalemia; muscle cramps, numbness \\
\hline $\begin{array}{l}\text { fast skeletal muscle troponin activators } \\
\text { Terasemtiv }\end{array}$ & improves muscle function & \\
\hline
\end{tabular}

\section{Myostatin and activin II receptor inhibitors}

Myostatin - the main inhibitor of muscle growth and satellite cell production. As myostatin activates the activin II receptor causing a signaling cascade through SMAD molecules, this group of intervention is known as "myostatin and activing II receptor inhibitors". Antibodies against myostatin were tested in the treatment of muscle wasting conditions and these are MYO-029, AMG 745, LY295655 and REGN1033, whereas the activing II receptor inhibitors are ACE011, ACE-031, ACE-083 and bimagrumab. Positive effects were obtained like enhancement of muscle mass, increased muscle fiber diameter, decreased fat mass, positive effects on muscle strength, but unfortunately also a lot of negative side effects were occurring which finally lead to a determination of using these compounds [6] ( Table 3).

\section{Mixed agonist/antagonist B1, B2, B3 activity, ACE inhibitors and fast skeletal muscle troponin activators}

Espindolol, is a non-specific mix of B1/B2 adrenergic receptor blocker that reduces catabolism and at the same time increases anabolism. It increases muscle mass and reduces fat mass. Perindopril is an angiotensin converting enzyme (ACE) inhibitor and seems to improve especially osteosarcopenic characteristics, whereas Terasemtiv is a fast skeletal muscle troponin activator which seems to improve muscle power [6].

\section{Classification of Sarcopenia}

In conclusion, we emphasize the detrimental outcome of sarcopenia in geriatric patients resulting in severe decrease of the patient's quality of life due to immobility, hospitalization and mortality. There are many etiopathophysiological cascades involved in the establishment of sarcopenia and many potential biomarkers and pharmaceutical targets are clinically approached. Up to now, the diagnostic algorithm is rather unspecific and is not able to differentiate between different causes. Consecutively the current treatment remains unspecific and is just based on improvement of nutrition and introduction of exercise. PFig. 2 summarizes our current ideas to search for novel diagnostic tools to classify the disease and to develop a mechanism-oriented therapy of sarcopenic patients with the help of distinct human models of geriatric sarcopenia. 


\section{Conflict of Interest}

No conflict of interest has been declared by the authors.

\section{Literature}

[1] Cruz-Jentoft AJ, Baeyens JP, Bauer JM et al. Sarcopenia: European consensus on definition and diagnosis: Report of the European Working Group on Sarcopenia in Older People. Age Ageing 2010; 39: $412-423$

[2] Rosenberg I. Summary comments: Epidemiological and methodological problems in determining nutritional status of older persons. Am J Clin Nutr 1987; 50: 1231-2133

[3] Morley JE, Abbatecola AM, Argiles JM et al. Sarcopenia with limited mobility: An international consensus. J Am Med Dir Assoc 2011; 12: 403-409

[4] Malmstrom TK, Miller DK, Simonsick EM et al. SARC-F: A symptom score to predict persons with sarcopenia at risk for poor functional outcomes. J Cachexia Sarcopenia Muscle 2016; 7: 28-36

[5] Woo J, Leung J, Morley JE. Validating the SARC-F: A suitable community screening tool for sarcopenia? J Am Med Dir Assoc 2014; 15: 630-634

[6] Morley JE. Pharmacologic Options for the Treatment of Sarcopenia. Calcif Tissue Int. Springer US 2016; 98: 319-333

[7] Morley JE, Argiles JM, Evans W] et al. Nutritional recommendations for the management of sarcopenia. J Am Med Dir Assoc 2010; p 391-396

[8] Anker SD, Morley JE, von Haehling S. Welcome to the ICD-10 code for sarcopenia. J Cachexia Sarcopenia Muscle 2016; 7: 512-514

[9] Koo BK, Roh E, Yang YS et al. Difference between old and young adults in contribution of $\beta$-cell function and sarcopenia in developing diabetes mellitus. J Diabetes Investig 2016; 7: 233-240

[10] Kalyani RR, Corriere M, Ferrucci L. Age-related and disease-related muscle loss: The effect of diabetes, obesity, and other diseases. Lancet Diabetes Endocrinol 2014; 2: 819-829

[11] Dimitriadis G, Mitrou P, Lambadiari V et al. Insulin effects in muscle and adipose tissue. Diabetes Res Clin Pract 2011; 93: S52-S59

[12] Cartee GD, Funai K. Exercise and insulin: Convergence or divergence at AS160 and TBC1D1? Exerc Sport Sci Rev 2009; 188-195

[13] Schiaffino S, Dyar KA, Ciciliot S et al. Mechanisms regulating skeletal muscle growth and atrophy. FEBS J 2013; 280: 4294-4314

[14] Cleasby ME, Jamieson PM, Atherton PJ. Insulin resistance and sarcopenia: Mechanistic links between common co-morbidities. J Endocrinol 2016; 229: R67-R81

[15] Cuthbertson DJ, Babraj ], Leese G et al. Anabolic resistance does not explain sarcopenia in patients with type 2 diabetes mellitus, compared with healthy controls, despite reduced mTOR pathway activity. Clin Nutr. Elsevier Ltd 2016; 10-13

[16] Lawrence JC. mTOR-dependent control of skeletal muscle protein synthesis. Int J Sport Nutr Exerc Metab 2001; 11 (Suppl): S177-S185

[17] Bouchi R, Fukuda T, Takeuchi T et al. Insulin Treatment Attenuates Decline of Muscle Mass in Japanese Patients with Type 2 Diabetes. Calcif Tissue Int. Springer US 2017; 0: 1-8

[18] Cetrone M, Mele A, Tricarico D. Effects of the antidiabetic drugs on the age-related atrophy and sarcopenia associated with diabetes type II. Curr Diabetes Rev 2014; 10: 231-237

[19] Rasmussen BB, Fujita $S$, Wolfe RR et al. Insulin resistance of muscle protein metabolism in aging. FASEB J. 2006; 20: 768-769
[20] Fujita S, Glynn EL, Timmerman KL et al. Supraphysiological hyperinsulinaemia is necessary to stimulate skeletal muscle protein anabolism in older adults: Evidence of a true age-related insulin resistance of muscle protein metabolism. Diabetologia 2009; 52: 1889-1898

[21] Wang X, Hu Z, Hu J et al. Insulin resistance accelerates muscle protein degradation: Activation of the ubiquitin-proteasome pathway by defects in muscle cell signaling. Endocrinology. 2006; 147: 4160-4168

[22] Shea MK, Nicklas BJ, Marsh AP et al. The effect of pioglitazone and resistance training on body composition in older men and women undergoing hypocaloric weight loss. Obesity (Silver Spring) 2011; 19: 1636-1646

[23] Zhou G, Myers R, Li Y et al. Role of AMP-activated protein kinase in mechanism of metformin action. J Clin Invest 2001; 108: 1167-1174

[24] Koh H], Brandauer ], Goodyear LJ. LKB1 and AMPK and the regulation of skeletal muscle metabolism. Curr Opin Clin Nutr Metab Care 2008; 11: 227-232

[25] Herzig S, Shaw RJ. AMPK: Guardian of metabolism and mitochondrial homeostasis. Nat. Rev. Mol. Cell Biol. 2018; 121-135

[26] Bujak AL, Crane JD, Lally JS et al. AMPK activation of muscle autophagy prevents fasting-induced hypoglycemia and myopathy during aging. Cell Metab. 2015; 21: 883-890

[27] Thomson DM, Gordon SE. Diminished overload-induced hypertrophy in aged fast-twitch skeletal muscle is associated with AMPK hyperphosphorylation. J Appl Physiol 2005; 98: 557-564

[28] Bolster DR, Crozier S], Kimball SR et al. AMP-activated protein kinase suppresses protein synthesis in rat skeletal muscle through down-regulated mammalian target of rapamycin (mTOR) signaling. J Biol Chem 2002; 277: 23977-23980

[29] Shan T, Zhang P, Liang X et al. Lkb1 is indispensable for skeletal muscle development, regeneration, and satellite cell homeostasis. Stem Cells. 2014; 32: 2893-2907

[30] Zhang C, McFarlane C, Lokireddy S et al. Myostatin-deficient mice exhibit reduced insulin resistance through activating the AMP-activated protein kinase signalling pathway. Diabetologia 2011; 54 : 1491-1501

[31] Das AK, Yang QY, Fu X et al. AMP-activated protein kinase stimulates myostatin expression in C2C12 cells. Biochem Biophys Res Commun 2012; 427: 36-40

[32] Sumantri S, Setiati S, Purnamasari D et al. Relationship between metformin and frailty syndrome in elderly people with type 2 diabetes. Acta Med Indones 2014; 46: 183-188

[33] Laksmi PW, Setiati S, Tamin TZ et al. Effect of metformin on handgrip strength, gait speed, myostatin serum level, and health-related quality of life: A double blind randomized controlled trial among non-diabetic pre-frail elderly patients. Acta Med Indones 2017; 49: 118-127

[34] Buteau J. GLP-1 receptor signaling: Effects on pancreatic beta-cell proliferation and survival. Diabetes Metab 2008; 34: (Suppl 2): S73-S77

[35] Nyström T. The potential beneficial role of glucagon-like peptide- 1 in endothelial dysfunction and heart failure associated with insulin resistance. Horm Metab Res 2008; 40: 593-606

[36] Chai W, Dong Z, Wang $\mathrm{N}$ et al. Glucagon-like peptide 1 recruits microvasculature and increases glucose use in muscle via a nitric oxide-dependent mechanism. Diabetes 2012; 61: 888-896

[37] Perna S, Guido D, Bologna C et al. Liraglutide and obesity in elderly: Efficacy in fat loss and safety in order to prevent sarcopenia. A perspective case series study. Aging Clin Exp Res 2016; 28 : $1251-1257$ 
[38] Giannocco G, Oliveira KC, Crajoinas RO et al. Dipeptidyl peptidase IV inhibition upregulates GLUT4 translocation and expression in heart and skeletal muscle of spontaneously hypertensive rats. Eur ] Pharmacol 2013; 698: 74-86

[39] Bouchi R, Fukuda T, Takeuchi T et al. Dipeptidyl peptidase 4 inhibitors attenuates the decline of skeletal muscle mass in patients with type 2 diabetes. Diabetes Metab Res Rev 2017

[40] Merovci A, Solis-Herrera C, Daniele G et al. Dapagliflozin improves muscle insulin sensitivity but enhances endogenous glucose production. J Clin Invest 2014; 124: 509-514

[41] Sugiyama S, Jinnouchi H, Kurinami N et al. Dapagliflozin reduces fat mass without affecting muscle mass in type 2 diabetes. J Atheroscler Thromb 2017; 25: 467-476

[42] Fujita S, Rasmussen BB, Cadenas JG et al. Aerobic exercise overcomes the age-related insulin resistance of muscle protein metabolism by improving endothelial function and akt / mammalian target of rapamycin signaling. Diabetes 2007; 56: 1615-1622

[43] Verreijen aM, Engberink MF, Verlaan S et al. Op019 exploration of what level of protein is needed for muscle mass accretion during weight loss with resistance training in obese elderly. Clin Nutr 2013; 32: 58

[44] Berr CM, Stieg MR, Deutschbein T et al. Persistence of myopathy in Cushing's syndrome: Evaluation of the German Cushing's Registry. Eur J Endocrinol 2017; 176: 737-746

[45] Drey M, Berr CM, Reincke M et al. Cushing's syndrome: A model for sarcopenic obesity. Endocrine 2017; 57: 481-485

[46] Kang S-H, Lee H-A, Kim M et al Forkhead box $\mathrm{O} 3$ plays a role in skeletal muscle atrophy through expression of $\mathrm{E} 3$ ubiquitin ligases MuRF-1 and atrogin-1 in Cushing's syndrome. Am J Physiol - Endocrinol Metab 2017; 312: E495-E507

[47] Zhao W, Qin W, Pan J et al. Dependence of dexamethasone-induced Akt/FOXO1 signaling, upregulation of MAFbx, and protein catabolism upon the glucocorticoid receptor. Biochem Biophys Res Commun. Elsevier Inc 2009; 378: 668-672

[48] Clarke BA, Drujan D, Willis MS et al. The E3 ligase MuRF1 degrades myosin heavy chain protein in dexamethasone-treated skeletal muscle. Cell Metab 2007; 6: 376-385

[49] Mammucari C, Milan G, Romanello $V$ et al. FoxO3 controls autophagy in skeletal muscle in vivo. Cell Metab 2007; 6: 458-471

[50] Baumgartner RN. Body composition in healthy aging. Ann N Y Acad Sci 2000; 904: 437-448

[51] Waters DL, Baumgartner RN, Garry P]. Advantages of dietary, exercise-related, and therapeutic interventions to prevent and treat sarcopenia in adult patients: An update. Clin Interv Aging 2010; 259-270

[52] Mazziotti G, Chiavistelli S, Giustina A. Pituitary diseases and bone. Endocrinol Metab Clin North Am 2015; 44: 171-180

[53] Ormsbee M], Prado CM, llich JZ et al. Osteosarcopenic obesity: the role of bone, muscle, and fat on health. J Cachexia Sarcopenia Muscle 2014; 5: 183-192

[54] Bolanowski M, Halupczok J, Jawiarczyk-Przybyłowska A. Pituitary disorders and osteoporosis. Int J Endocrinol 2015, doi:10.1155/ 2015/206853

[55] Doak CM, Wijnhoven TMA, Schokker DF et al. Age standardization in mapping adult overweight and obesity trends in the WHO European Region. Obes Rev 2012; 174-191

[56] Fahal IH. Uraemic sarcopenia: Aetiology and implications. Nephrol Dial Transplant 2014; 29: 1655-1665

[57] Epstein FH, Mitch WE, Goldberg AL. Mechanisms of muscle wasting - the role of the ubiquitin-proteasome pathway. N Engl J Med 1996; 335: 1897-1905
[58] Kaizu Y, Ohkawa S, Odamaki M et al. Association between inflammatory mediators and muscle mass in long-term hemodialysis patients. Am J Kidney Dis 2003; 42: 295-302

[59] Sato E, Mori T, Mishima E et al. Metabolic alterations by indoxyl sulfate in skeletal muscle induce uremic sarcopenia in chronic kidney disease. Sci Rep 2016; 6: 36618

[60] Song YH, Li Y, Du J et al. Muscle-specific expression of IGF-1 blocks angiotensin II-induced skeletal muscle wasting. J Clin Invest 2005; 115: 451-458

[61] Delafontaine P, Yoshida T. The renin-angiotensin system and the biology of skeletal muscle: Mechanisms of muscle wasting in chronic disease states. Trans Am Clin Climatol Assoc 2016; 127: 245-258

[62] Montano-Loza AJ, Angulo P, Meza-Junco J et al. Sarcopenic obesity and myosteatosis are associated with higher mortality in patients with cirrhosis. J Cachexia Sarcopenia Muscle 2015; 6: 126-135

[63] Stumvoll M, Meyer C, Mitrakou A et al. Important role of the kidney in human carbohydrate metabolism. Med Hypotheses 1999; 52: 363-366

[64] Wang XH, Du J, Klein JD et al. Exercise ameliorates chronic kidney disease-induced defects in muscle protein metabolism and progenitor cell function. Kidney Int 2009; 76: 751-759

[65] Caballero PEJ. Klinefelter's syndrome associated with progressive muscular atrophy simulating Kennedy's disease. Ann Indian Acad Neurol 2012; 15: 227-229

[66] Bardsley MZ, Falkner B, Kowal K et al. Insulin resistance and metabolic syndrome in prepubertal boys with Klinefelter syndrome. Acta Paediatr 2011; 100: 866-870

[67] Basualto-Alarcón C, Varela D, Duran J et al. Sarcopenia and androgens: A link between pathology and treatment. Front Endocrinol (Lausanne) 2014; 5: 217

[68] Perry HM, Horowitz M, Morley JE et al. Longitudinal changes in serum 25-hydroxyvitamin D in older people. Metabolism 1999; 48 : 1028-1032

[69] Feldman HA, Longcope C, Derby CA et al. Age trends in the level of serum testosterone and other hormones in middle-aged men: Longitudinal results from the Massachusetts Male Aging Study. J Clin Endocrinol Metab 2002; 87: 589-598

[70] Nieschlag E. Current topics in testosterone replacement of hypogonadal men. Best Pract Res Clin Endocrinol Metab 2015; 29 : 77-90

[71] Frisoli A, Chaves PHM, Pinheiro MM et al. The effect of nandrolone decanoate on bone mineral density, muscle mass, and hemoglobin levels in elderly women with osteoporosis: A double-blind, randomized, placebo-controlled clinical trial. J Gerontol A Biol Sci Med Sci 2005; 60: 648-653

[72] Chiò A, Logroscino G, Traynor B] et al. Global epidemiology of amyotrophic lateral sclerosis: A systematic review of the published literature. Neuroepidemiology 2013; 41: 118-130

[73] Haverkamp LJ, Appel V, Appel SH. Natural history of amyotrophic lateral sclerosis in a database population. Validation of a scoring system and a model for survival prediction. Brain 1995; 118 (Pt 3): 707-719

[74] Chia R, Chiò A, Traynor B]. Novel genes associated with amyotrophic lateral sclerosis: Diagnostic and clinical implications. Lancet Neurol 2018; 17: 91-102

[75] Cappello V, Francolini M. Neuromuscular junction dismantling in amyotrophic lateral sclerosis. Int J Mol Sci 2017; doi:10.3390/ ijms18102092

[76] Moloney EB, de Winter F, Verhaagen J. ALS as a distal axonopathy: Molecular mechanisms affecting neuromuscular junction stability in the presymptomatic stages of the disease. Front Neurosci 2014; 8: 252 
[77] Uitti R], Berry K, Yasuhara O et al. Neurodegenerative 'overlap' syndrome: Clinical and pathological features of Parkinson's disease, motor neuron disease, and Alzheimer's disease. Parkinsonism Relat Disord 1995; 1: 21-34

[78] Drey M, Hasmann SE, Krenovsky J-P et al. Associations between Early Markers of Parkinson's Disease and Sarcopenia. Front Aging Neurosci 2017; 9: 53

[79] Bromberg MB. MUNIX and MUNE in ALS. Clin Neurophysiol. 2013; 124: $433-434$

[80] McComas A], Fawcett PR, Campbell M] et al. Electrophysiological estimation of the number of motor units within a human muscle. J Neurol Neurosurg Psychiatry 1971; 34: 121-131

[81] Nandedkar SD, Nandedkar DS, Barkhaus PE et al. Motor unit number index (MUNIX). IEEE Trans Biomed Eng 2004; 51: 2209-2211

[82] Neuwirth C, Nandedkar S, Stålberg E et al. Motor Unit Number Index (MUNIX): A novel neurophysiological marker for neuromuscular disorders; test-retest reliability in healthy volunteers. Clin Neurophysiol. International Federation of Clinical Neurophysiology 2011 122: $1867-1872$

[83] Nandedkar SD, Barkhaus PE, Stålberg EV. Reproducibility of MUNIX in patients with amyotrophic lateral sclerosis. Muscle Nerve. 2011; 44: 919-922

[84] Drey M, Grösch C, Neuwirth C et al. The Motor Unit Number Index (MUNIX) in sarcopenic patients. Exp Gerontol 2013; 48: 381-384

[85] Drey M, Krieger B, Sieber CC et al. Motoneuron loss is associated with sarcopenia. J Am Med Dir Assoc 2014; 15: 435-439

[86] Rudolf R, Deschenes MR, Sandri M. Neuromuscular junction degeneration in muscle wasting. Curr Opin Clin Nutr Metab Care 2016; 19: 177-181

[87] Stephan A, Mateos JM, Kozlov SV et al. Neurotrypsin cleaves agrin locally at the synapse. FASEB J 2008; 22: 1861-1873

[88] Drey M, Sieber CC, Bauer JM et al. C-terminal Agrin Fragment as a potential marker for sarcopenia caused by degeneration of the neuromuscular junction. Exp Gerontol 2013; 48: 76-80
89] Hettwer S, Lin S, Kucsera S et al. Injection of a soluble fragment of neural agrin (NT-1654) considerably improves the muscle pathology caused by the disassembly of the neuromuscular junction. PLoS One 2014; 9: e88739

[90] Heymsfield SB, Adamek M, Gonzalez MC et al. Assessing skeletal muscle mass: Historical overview and state of the art. J Cachexia Sarcopenia Muscle 2014; 9-18

[91] Scharf G, Heineke J. Finding good biomarkers for sarcopenia. J Cachexia Sarcopenia Muscle 2012; 3: 145-148

[92] Ebner N, Steinbeck L, Doehner W et al. Highlights from the 7th Cachexia Conference: Muscle wasting pathophysiological detection and novel treatment strategies. J Cachexia Sarcopenia Muscle 2014; 5: $27-34$

[93] Drescher C, Konishi M, Ebner N et al. Loss of muscle mass: Current developments in cachexia and sarcopenia focused on biomarkers and treatment. Int J Cardiol 2016; 202: 766-772

94] Arnold AS, Egger A, Handschin C. PGC- $1 \alpha$ and myokines in the aging muscle - A mini-review. Gerontology 2010; 37-43

[95] Inui A. Cancer anorexia-cachexia syndrome: Current issues in research and management. CA Cancer J Clin 2002; 52: 72-91

[96] Morley JE, Kaiser FE, Perry HM et al. Longitudinal changes in testosterone, luteinizing hormone, and follicle-stimulating hormone in healthy older men. Metabolism 1997; 46: 410-413

[97] Morley JE. Scientific overview of hormone treatment used for rejuvenation. Fertil Steril 2013; 1807-1813

[98] Kovacheva EL, Sinha Hikim AP et al. Testosterone supplementation reverses sarcopenia in aging through regulation of myostatin, c-Jun $\mathrm{NH} 2$-terminal kinase, Notch, and Akt signaling pathways. Endocrinology. 2010; 151: 628-638

[99] Crawford JC, Johnston MA, Hancock ML et al. Enobosarm, a selective androgen receptor modulator (SARM), increases lean bodymass (LBM) in advanced nsclc patients; updated results of two pivotal, international phase 3 trials. Support Care Cancer 2014; 22: S30-S31 